\title{
Second-quantized Landau-Zener theory for dynamical instabilities
}

\author{
J.R. Anglin \\ Center for Ultracold Atoms, Massachusetts Institute of Technology, 77 Massachusetts Ave., Cambridge MA 02139
}

(Dated: June 29, 2021)

\begin{abstract}
State engineering in nonlinear quantum dynamics sometimes may demand driving the system through a sequence of dynamically unstable intermediate states. This very general scenario is especially relevant to dilute Bose-Einstein condensates, for which ambitious control schemes have been based on the powerful Gross-Pitaevskii mean field theory. Since this theory breaks down on logarithmically short time scales in the presence of dynamical instabilities, an interval of instabilities introduces quantum corrections, which may possibly derail a control scheme. To provide a widely applicable theory for such quantum corrections, this paper solves a general problem of time-dependent quantum mechanical dynamical instability, by modelling it as a second-quantized analogue of a Landau-Zener avoided crossing: a 'twisted crossing'.
\end{abstract}

Quantum dynamical instabilities (complex excitation frequencies) have been associated with formation of solitons and vortices in driven Bose-Einstein condensates. For example, in Refs. [1] and [2], the Bogoliubov spectra are examined for two-parameter families of mean field states. Although the physics involved is significantly different (one-dimensional modulated current versus rotating harmonic trap), figures in both these works show dynamical instabilities in narrow, finger-like regions of parameter space (see Figure 1). We will show below that this is generic for dynamically unstable quasiparticle excitations in weakly interacting many-body systems.

Such systems, especially condensates, may be sufficiently weakly damped that state engineering under energetic instability becomes feasible. Interesting structures such as solitons and sonic event horizons will require this capability. But to access the entire volume of energetically unstable control space, it will also be a common task to drive systems briefly through narrow regions of dynamical instability. Mean field theory and adiabatic approximations, which are important tools of state engineering, will break down during these episodes [3]. This paper therefore exploits an analogy between dynamical instability in second quantization, and avoided crossing in two-state quantum mechanics [4], to determine the quantum effects of driving a system through dynamical instability. An almost identical calculation has recently been presented [5] to deal with the specific dynamical instability involved in dissociation of a molecular condensate [6]; the present paper provides an explicit derivation of the result in a general context.

Quantum dynamical instability is not always recognized as a possibility, because with a Hermitian Hamiltonian, the eigenfrequencies of Schrödinger evolution must all be real. But the eigenfrequencies of Heisenberg evolution may be complex. It is easy to see this for the manifestly Hermitian Hamiltonian

$$
\begin{aligned}
\hat{H}= & (\omega+\delta) \hat{a}_{+}^{\dagger} \hat{a}_{+}+(\delta-\omega) \hat{a}_{-}^{\dagger} \hat{a}_{-} \\
& +\gamma\left(\hat{a}_{+}^{\dagger} \hat{a}_{-}^{\dagger}+\hat{a}_{+} \hat{a}_{-}\right)
\end{aligned}
$$

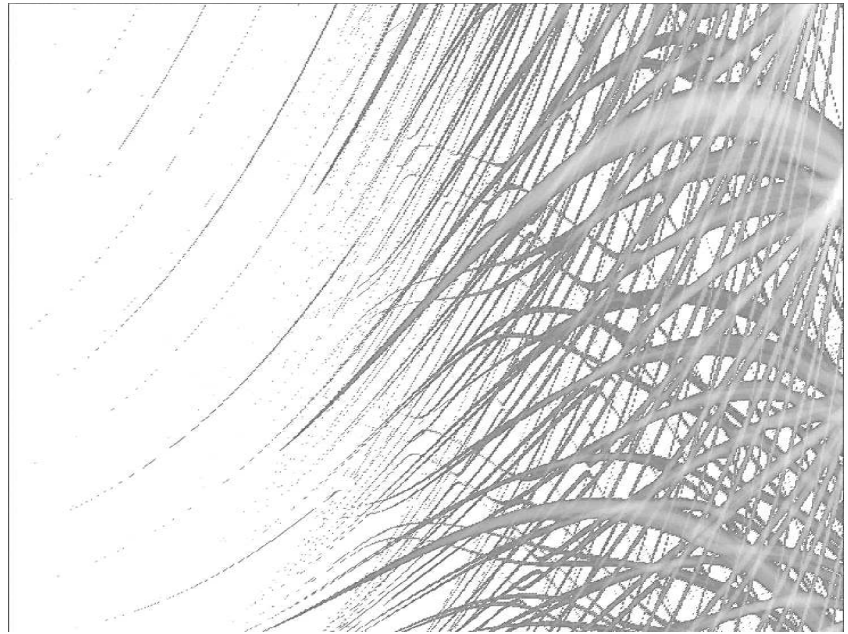

FIG. 1: Grayscale plot of sum of absolute values of imaginary parts of Bogoliubov excitation frequencies for a quasione-dimensional condensate flowing with winding number 10 around a toroidal trap with modulated potential. Axes are control parameters: mean condensate density increases from bottom to top, severity of density modulation increases from left to right. Compare to figures in Refs. [1], 2].

where all co-efficients are real, and $\hat{a}_{ \pm}$and $\hat{a}_{ \pm}^{\dagger}$ are ordinary annihilation and creation operators (i.e. $\left[\hat{a}_{j}, \hat{a}_{k}^{\dagger}\right]=$ $\delta_{j k}$ is the only non-vanishing commutator). The eigenoperators of Heisenberg evolution satisfy

$$
\begin{aligned}
i \dot{\hat{b}}_{ \pm} & =\left[\hat{b}_{ \pm}, \hat{H}\right]=\Omega_{ \pm} \hat{b}_{ \pm} \\
\hat{b}_{ \pm} & =\frac{\left(\delta \pm \sqrt{\delta^{2}-\gamma^{2}}\right)^{\frac{1}{2}} \hat{a}_{+}+\left(\delta \mp \sqrt{\delta^{2}-\gamma^{2}}\right)^{\frac{1}{2}} \hat{a}_{-}^{\dagger}}{\sqrt{2}\left|\delta^{2}-\gamma^{2}\right|^{1 / 4}} \\
\Omega_{ \pm} & =\omega \pm \sqrt{\delta^{2}-\gamma^{2}}
\end{aligned}
$$

where we assume $\gamma \geq 0$ (without loss of generality, since we can always redefine $\left.\hat{a}_{+} \rightarrow-\hat{a}_{+}\right)$. These operators always obey $\left[\hat{b}_{+}, \hat{b}_{-}\right]=0$. As long as $\gamma<|\delta|, \Omega_{ \pm}$are both real, and we also have

$$
\gamma<|\delta|: \quad\left[\hat{b}_{ \pm}, \hat{b}_{ \pm}^{\dagger}\right]= \pm \operatorname{sgn}(\delta) \quad, \quad\left[\hat{b}_{ \pm}, \hat{b}_{\mp}^{\dagger}\right]=0 .
$$


And we can write

$$
\hat{H}=\operatorname{sgn}(\delta) \times\left[E_{0}+\sum_{ \pm} \pm \Omega_{ \pm} \hat{b}_{ \pm}^{\dagger} \hat{b}_{ \pm}\right], \quad \gamma<|\delta|
$$

for $E_{0}=\delta-\omega$. This means that as long as $\gamma<|\delta|$, one of the $\hat{b}_{ \pm}$is really a creation operator which would have a ${ }^{\dagger}$ in more conventional notation; but apart from this trivial issue of labelling, we have diagonalized to noninteracting quasiparticles of the standard (bosonic) kind.

If $\gamma>|\delta|$, however, $\Omega_{ \pm}$are complex, and neither $\hat{b}_{ \pm}$ nor $\hat{b}_{ \pm}^{\dagger}$ are standard annihilation operators. They obey

$$
\gamma>|\delta|: \quad\left[\hat{b}_{ \pm}, \hat{b}_{ \pm}^{\dagger}\right]=0 \quad, \quad\left[\hat{b}_{ \pm}, \hat{b}_{\mp}^{\dagger}\right]= \pm i
$$

so that we must say that the canonical conjugate of $\hat{b}_{ \pm}$ is $\mp i \hat{b}_{\mp}^{\dagger}$, and no longer coincides with the Hermitian conjugate. In this case we have

$$
\hat{H}=E_{0}+\sum_{ \pm} \mp i \Omega_{ \pm} \hat{b}_{\mp}^{\dagger} \hat{b}_{ \pm}, \quad \gamma>|\delta|
$$

instead of (6). One can also show that if $\gamma>|\delta|$ then the eigenvalues of $\hat{H}$ no longer involve integer multiples of $\Omega_{ \pm}$: they remain real, but are continuous. Hence in this case there is no way to interpret the energy eigenstates as having definite numbers of any kind of quasiparticle.

If $\gamma>|\delta|$, then $\hat{H}$ is unbounded below as well as above; this can also occur even for $\gamma<|\delta|$, if $|\omega|>\sqrt{\delta^{2}-\gamma^{2}}$. In these cases, (11) can be physical only as the linearization of a nonlinear Hamiltonian about an excited state. And in all cases, since the 'anomalous coupling' term proportional to $\gamma$ in (11) does not conserve the numbers of particles created and annihilated by $\hat{a}_{ \pm}^{\dagger}$ and $\hat{a}_{ \pm}$, these must be some kind of quasiparticle, or else there must be a source of atoms (such as a molecular condensate treated as a c-number field (5)). But there are many realistic situations in which unboundedness of the linearized Hamiltonian, and non-conservation of (quasi-)particles, are perfectly correct. For these (11) is universal, in the sense that for all eigenfrequencies, whether real or complex, pairs of modes may be expressed in this form by using (3) to define $\hat{a}_{ \pm}$.

Two-body interactions among particles lead to quasiparticle Hamiltonians with anomalous couplings as in (1). Since $\gamma$ is thus constrained to be small if interactions are weak, dynamical instabilities tend to arise in weakly interacting systems not through $\gamma$ becoming large, but through $\delta$ becoming even smaller. A dynamical instability is thus a kind of second-quantized analogue to an avoided crossing [7]. See Figure 2. If the many-body Hamiltonian depends on some number $D$ of control parameters, then any two modes will have frequencies exactly opposite on a $(D-1)$-dimensional parameter subspace, and so there will be a dynamical instability within a thin shell around that (hyper)surface. Of course, dynamical instabilities can only arise if there is energetic
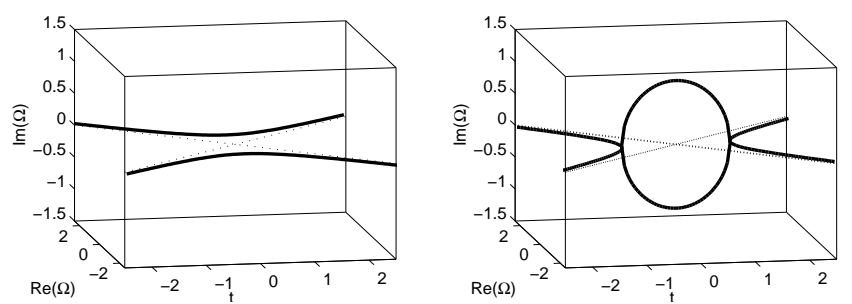

FIG. 2: At left, an ordinary avoided crossing; at right, a 'twisted crossing' with complex intermediate eigenfrequencies.

instability, so that some negative frequency modes exist. But if the system is energetically unstable, then fingerlike (or in higher dimensioned control spaces, hypershelllike) regions of dynamical instability are generic.

Since much state engineering can be accomplished by means of time-dependent control parameters in the Hamiltonian, we need to assess the effects of a time dependence that brings a system through an intermediate interval in which one dynamical instability exists. Focusing our attention on the pair of modes involved in the instability, we can always cast them in the form of (1)), with time-dependent $\omega, \delta, \gamma$. As long as $|\delta| \gg \gamma$ we can apply standard methods, but a connection formula is required to give the quantum evolution over the intermediate interval. Within this interval, we assume the widely applicable case that $\omega$ and $\gamma$ are essentially constant, and $\delta$ changes linearly with time. Since $\left(\hat{a}_{+}^{\dagger} \hat{a}_{+}-\hat{a}_{-}^{\dagger} \hat{a}_{-}\right)$ commutes with $\hat{H}$, we can eliminate $\omega$ in an interaction picture by taking $\hat{a}_{ \pm} \rightarrow e^{\mp i \omega t} \hat{a}_{ \pm}$, giving us the problem

$$
\hat{H}_{i}=\gamma\left(\hat{a}_{+}^{\dagger} \hat{a}_{-}^{\dagger}+\hat{a}_{+} \hat{a}_{-}\right)+\zeta^{2} t\left(\hat{a}_{+}^{\dagger} \hat{a}_{+}+\hat{a}_{-}^{\dagger} \hat{a}_{-}\right)
$$

for a constant rate $\zeta$.

By examining the Heisenberg equations of motion with this time-dependent Hamiltonian we can straightforwardly obtain

$$
\begin{aligned}
& \lim _{t \rightarrow-\infty} \hat{a}_{ \pm}(t)=e^{-i \theta(t)} \hat{a}_{ \pm I N}-\frac{\gamma e^{i \theta(t)}}{2 \zeta^{2} t} \hat{a}_{\mp I N}^{\dagger} \\
& \lim _{t \rightarrow+\infty} \hat{a}_{ \pm}(t)=e^{-i \theta(t)} \hat{a}_{ \pm} \text {OUT }-\frac{\gamma e^{i \theta(t)}}{2 \zeta^{2} t} \hat{a}_{\mp}^{\dagger} \text { OUT }
\end{aligned}
$$

where

$$
\theta(t)=\frac{\zeta^{2} t^{2}}{2}-\frac{\gamma^{2}}{2 \zeta^{2}} \ln |2 \zeta t|+\mathcal{O}\left(t^{-2}\right)
$$

and $\hat{a}_{ \pm I N, O U T}$ are time-independent annihilation operators, whose relationship to each other unfortunately depends on the intervening evolution at finite times. (The factor multiplying $t$ in the argument of the logarithm in (12) is not actually fixed by the equation of motion, but is chosen for our notational convenience below.) Of course the point of the limits $t \rightarrow \pm \infty$ is not literally to consider infinite times, but only to give the asymptotic behaviour 
which can be smoothly matched to adiabatic approximations valid for $\zeta^{2}|t| \gg \gamma$. Hence in the Heisenberg picture no further definition of $\hat{a}_{ \pm I N}$ is required: it will be given in terms of initial time operators by solving the evolution problem for $t \ll-\zeta^{-2} \gamma$. So the problem is to determine the OUT operators in terms of the $I N$ operators.

This problem is directly analogous to the LandauZener problem for amplitudes in a two-state system being driven through an avoided crossing, except that the amplitudes are replaced by operators, and the secondquantized problem allows the 'anomalous' coupling which would be non-Hermitian in the two-state system. Because our problem is also linear, we can solve it in a very similar way. Iterating the Heisenberg equations to obtain a second order equation for $\hat{a}_{+}$with $\hat{a}_{-}^{\dagger}$ eliminated,

$$
\left[\partial_{t t}+2 i \zeta^{2} t \partial_{t}-\gamma^{2}+2 i \zeta^{2}\right]\left(e^{-i \frac{\zeta^{2} t^{2}}{2}} \hat{a}_{+}\right)=0
$$

then Fourier transforming from $t$ to frequency $\nu$, we find an easily-solved first order differential equation in $\nu$ :

$$
\begin{aligned}
\hat{a}_{+}(t) & \equiv e^{i \frac{\zeta^{2} t^{2}}{2}} \int d \nu e^{-i \nu t} \hat{\alpha}(\nu) \\
\partial_{\nu} \hat{\alpha} & =-\frac{\left(\nu^{2}+\gamma^{2}\right)}{2 i \zeta^{2} \nu} \hat{\alpha} .
\end{aligned}
$$

Inverse Fourier transforming back to $t$ then gives

$$
\begin{aligned}
\hat{a}_{+}(t) & =\sum_{j=1,2} \hat{\alpha}_{j} A_{j}(t) \\
A_{j}(t) & \equiv e^{i \frac{\zeta^{2} t^{2}}{2}} \int_{C_{j}} \frac{d \nu}{\zeta} e^{i S_{t}(\nu)} \\
S_{t}(\nu) & \equiv-\nu t+\frac{\nu^{2}}{4 \zeta^{2}}+\frac{\gamma^{2}}{2 \zeta^{2}} \ln \frac{\nu}{\zeta} .
\end{aligned}
$$

There are two independent solutions $A_{1,2}(t)$, with independent operator-valued co-efficients $\hat{\alpha}_{1,2}$, since the $\nu$ integral may be evaluated along inequivalent contours $C_{1,2}$ in the complex $\nu$-plane. Since the integrand vanishes at the branch point $\nu=0$, and at infinity between angles $\left(0, \frac{\pi}{2}\right)$ and $\left(\pi, \frac{3 \pi}{2}\right)$, integration contours can only terminate at these points (because terminating anywhere else amounts to replacing the integrand with something that does vanish elsewhere). The simplest distinct contours are $C_{1}$ running from lower-left infinity to upper-right, and $C_{2}$ running from zero to upper-right infinity. Since the integrand is analytic except at upper-left and lowerright infinity, and on the branch cut (which we take to run along the negative imaginary axis), we are free to choose contours that make the evaluation easier; see Fig. 3.

A given choice of contour yields a single solution valid for all $t$, but for $|t| \rightarrow \infty$ the integral may be evaluated using combinations of the method of steepest descents and integral representations of the Gamma function. (There is a second saddlepoint near the origin, but as $|t| \rightarrow \infty$ it
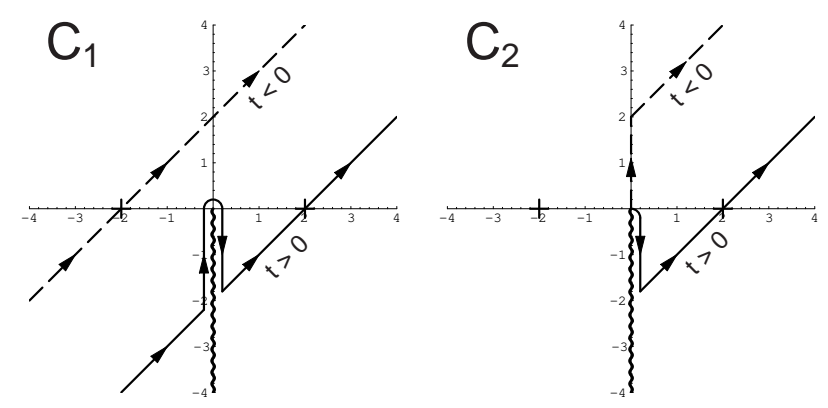

FIG. 3: Contours used in the text, for $\zeta^{2} t \gg \gamma$ (solid) and $\zeta^{2} t \ll-\gamma$ (dashed), plotted in the complex plane of $\nu /\left(\zeta^{2}|t|\right)$. Saddlepoints are marked with crosses on the real axis at $2 \operatorname{sgn}(t)$. At left, contour $C_{1}$ traverses from lower-left to upper-right infinity; at right, contour $C_{2}$ runs from 0 to upper-right infinity.

is too close to the branch point for the method of steepest descents to work, and so contour $C_{2}$ must run along the imaginary axis until it reaches a contour of steepest descent through the other saddlepoint.) The asymptotic behaviours of the full solutions $A_{j}(t)$ can thus be obtained explicitly, allowing us to determine the late time behaviour of the solutions whose early time behaviour matches (10).

The contributions to the contour integrals for $A_{j}(t)$ from the diagonal portions are dominated by the saddlepoints $\nu_{t}=2 \zeta^{2} t+\mathcal{O}\left(\frac{\gamma^{2}}{\zeta^{2} t}\right)$, since for $\nu=2 \zeta^{2} t+e^{i \pi / 4} \zeta s$,

$$
\begin{aligned}
\int \frac{d \nu}{\zeta} e^{i S}= & \int d s e^{-\frac{1}{4} s^{2}}\left[1+\frac{i \gamma^{2} e^{i \frac{\pi}{4}} s}{2 \zeta^{3} t}+\mathcal{O}\left(\frac{s^{2}}{\zeta^{2} t^{2}}\right)\right] \\
& \times e^{-i\left(\zeta^{2} t^{2}-\frac{\gamma^{2}}{2 \zeta^{2}} \ln (2 \zeta t)-\frac{\pi}{4}\right)}
\end{aligned}
$$

So if the contour passes through the saddlepoint we get a contribution to $A_{j}(t)$

$$
\begin{aligned}
{\left[1+\mathcal{O}(\zeta t)^{-2}\right] A_{S P}=} & 2 \sqrt{\pi} e^{i \frac{\pi}{4}} e^{-i\left(\frac{\zeta^{2} t^{2}}{2}-\frac{\gamma^{2}}{2 \zeta^{2}} \ln |2 \zeta t|\right)} \\
& \times\left\{\begin{aligned}
1 & , t>0 \\
e^{-\frac{\pi \gamma^{2}}{2 \zeta^{2}}} & , t<0
\end{aligned}\right.
\end{aligned}
$$

If a diagonal segment shown in Fig. 3 does not cross a saddlepoint, then this segment yields only an utterly negligible contribution $\sim e^{-2 \zeta^{2} t^{2}}$.

For the vertical segments along the imaginary axis in $C_{2}$, we let $\nu=-i \xi / t+0$ and integrate

$$
\begin{aligned}
\int \frac{d \nu}{\zeta} e^{i S_{t}} & =\frac{1}{i \zeta t} \int_{0}^{2(\zeta t)^{2}} d \xi e^{-\xi+i \frac{\gamma^{2}}{2 \zeta^{2}} \ln \frac{0-i \xi}{\zeta t}} e^{-i \frac{\xi^{2}}{4 \zeta^{2} t^{2}}} \\
& \doteq \frac{e^{-i \frac{\gamma^{2}}{2 \zeta^{2}} \ln |\zeta t|} e^{\frac{\pi \gamma^{2}}{4 \zeta^{2}} \operatorname{sgn}(t)}}{i \zeta t} \int_{0}^{\infty} d \xi \xi^{\frac{i \gamma^{2}}{2 \zeta^{2}}} e^{-\xi} \\
& =\frac{e^{-i \frac{\gamma^{2}}{2 \zeta^{2}} \ln |\zeta t|} e^{\frac{\pi \gamma^{2}}{4 \zeta^{2}} \operatorname{sgn}(t)}}{i \zeta t} \Gamma\left(1+\frac{i \gamma^{2}}{2 \zeta^{2}}\right)
\end{aligned}
$$


where in the second line we extend the integration limit to infinity by ignoring terms of order $e^{-2(\zeta t)^{2}}$, and drop the $\exp -i \frac{\xi^{2}}{4 \zeta^{2} t^{2}}$ from the integrand because it yields corrections of order $(\zeta t)^{-2}$. This yields the contribution

$$
\begin{aligned}
A_{I M}= & \frac{2^{i \frac{\gamma^{2}}{2 \zeta^{2}}}}{i \zeta t} e^{\frac{\pi \gamma^{2}}{4 \zeta^{2}} \operatorname{sgn}(t)} \Gamma\left(1+\frac{i \gamma^{2}}{2 \zeta^{2}}\right) e^{i\left[\frac{\zeta^{2} t^{2}}{2}-\frac{\gamma^{2}}{2 \zeta^{2}} \ln |2 \zeta t|\right]} \\
& \times\left[1+\mathcal{O}(\zeta t)^{-2}\right]
\end{aligned}
$$

From the two segments at $t>0$ where $C_{1}$ hugs the branch cut, we gain a contribution $\left(1-e^{-\frac{\pi \gamma^{2}}{\zeta^{2}}}\right) A_{I M}$.

Combining these contributions and recalling our definition of $\theta(t)$ in (12), we obtain, up to corrections of order $(\zeta t)^{-2}$,

$$
\begin{aligned}
& A_{1}(t)=\left\{\begin{array}{cc}
2 \sqrt{\pi} e^{i \frac{\pi}{4}} e^{-i \theta(t)} e^{-\frac{\pi \gamma^{2}}{2 \zeta^{2}}}, \quad t<0 \\
2 \sqrt{\pi} e^{i \frac{\pi}{4}} e^{-i \theta(t)}+\mathcal{O}(\zeta t)^{-1}, t>0
\end{array}\right. \\
& A_{2}(t)=\left\{\begin{array}{cc}
\frac{2^{i \frac{\gamma^{2}}{2 \zeta^{2}}}}{i \zeta t} e^{-\frac{\pi \gamma^{2}}{4 \zeta^{2}}} e^{i \theta(t)} \Gamma\left(1+\frac{i \gamma^{2}}{2 \zeta^{2}}\right), & t<0 \\
2 \sqrt{\pi} e^{i \frac{\pi}{4}} e^{-i \theta(t)}+\mathcal{O}(\zeta t)^{-1} & , t>0
\end{array}\right.
\end{aligned}
$$

where the suppressed $\mathcal{O}(\zeta t)^{-1}$ terms can easily be computed from (19), but will not be needed.

Comparing (20) with (10) and (15), we can read off

$$
\begin{aligned}
\hat{\alpha}_{1} & =\frac{e^{-i \frac{\pi}{4}} e^{\frac{\pi \gamma^{2}}{2 \zeta^{2}}}}{2 \sqrt{\pi}} \hat{a}_{+I N} \\
\hat{\alpha}_{2} & =\frac{2^{-i \frac{\gamma^{2}}{2 \zeta^{2}} i \gamma}}{2 \zeta} \frac{e^{\frac{\pi \gamma^{2}}{4 \zeta^{2}}}}{\Gamma\left(1+\frac{i \gamma^{2}}{2 \zeta^{2}}\right)} \hat{a}_{-I N}^{\dagger} \\
& =\frac{2^{-i \frac{\gamma^{2}}{2 \zeta^{2}}}}{2 i \sqrt{\pi}}\left[\frac{\Gamma\left(1-\frac{i \gamma^{2}}{2 \zeta^{2}}\right)}{\Gamma\left(1+\frac{i \gamma^{2}}{2 \zeta^{2}}\right)}\right]^{\frac{1}{2}}\left[e^{\frac{\pi \gamma^{2}}{\zeta^{2}}}-1\right]^{\frac{1}{2}} \hat{a}_{-I N}^{\dagger}
\end{aligned}
$$

using the $\Gamma$-function identity

$$
|\Gamma(1+i x)|^{2}=\frac{\pi x}{\sinh \pi x} .
$$

Then comparing these last results with (11), and noting that the equations for $\hat{a}_{-}$and $\hat{a}_{+}^{\dagger}$ are exactly similar to those we have examined, we finally conclude

$$
\begin{aligned}
& \hat{a}_{ \pm} O U T=e^{\frac{\pi}{2} \gamma^{2} / \zeta^{2}} \hat{a}_{ \pm I N}+e^{i \pi}\left[e^{\pi / / \zeta^{2}}-1\right]^{\frac{1}{2}} \hat{a}_{\mp I N}^{\dagger} \\
& e^{i \eta}=2^{-i \frac{\gamma^{2}}{2 \zeta^{2}}} e^{-i \frac{\pi}{4}}\left[\frac{\Gamma\left(1-\frac{i \gamma^{2}}{2 \zeta^{2}}\right)}{\Gamma\left(1+\frac{i \gamma^{2}}{2 \zeta^{2}}\right)}\right]^{\frac{1}{2}} .
\end{aligned}
$$

Equation 25) is our main result. It is indeed very similar to the Landau-Zener formula, except for the vital difference that the exponents are all positive rather than negative. Consequently, in the state which is annihilated by $\hat{a}_{ \pm I N}$, the expected numbers of quasiparticles after passing through the instability will be

$$
\left\langle 0_{I N}\left|\hat{a}_{ \pm O U T}^{\dagger} \hat{a}_{ \pm O U T}\right| 0_{I N}\right\rangle=e^{\frac{\pi \gamma^{2}}{\zeta^{2}}}-1
$$

Driving through a dynamical instability in less than the characteristic time scale of the instability produces only mild excitation, but exceeding this time generates quasiparticles explosively. And the crossover between these regimes is rather abrupt. This may set important practical limits to the precision of state engineering, because controlling faster than $\gamma$ may mean not being slow enough to be adiabatic for other, dynamically stable modes of the total nonlinear system.

Note that changing $\zeta^{2} \rightarrow-\zeta^{2}$ in (9), so that the system is stable at early times rather than late, produces only $e^{i \eta} \rightarrow-e^{-i \eta}$ in 25. And while we have analyzed the most general type of dynamical instability, with complex frequency and two degrees of freedom involved, the simpler case with purely imaginary frequency, which was treated in [5], can be obtained by setting $\omega \rightarrow 0$, and $\hat{a}_{ \pm} \rightarrow \hat{a} / \sqrt{2}$ in (11). Everywhere else, one must use simply $\hat{a}_{ \pm} \rightarrow \hat{a}$ instead; there are also trivial changes to (6) and (8). We have therefore obtained a quite general result that will be useful for state engineering through dynamical instabilities.

[1] L.J. Garay, J.R. Anglin, J.I. Cirac and P. Zoller, Phys. Rev. Lett. 85, 4643 (2000); Phys. Rev. A63, 023611 (2001).

[2] S. Sinha and Y. Castin, Phys. Rev. Lett. 87, 190402 (2001).

[3] A. Vardi and J.R. Anglin, Phys. Rev. Lett. 86, 568 (2001); J.R. Anglin and A. Vardi, Phys. Rev. A64, 013605 (2001).

[4] L.D. Landau, Phys. Z. Sowjetunion 2, 46 (1932); C. Zener, Proc. R. Soc. London A 137, 696 (1932).

[5] V.A. Yurovsky, A. Ben-Reuven, and P.S. Julienne, condmat/0108372.

[6] A. Vardi, V.A. Yurovsky and J.R. Anglin, Phys. Rev. A64, 063611 (2001).

[7] D.V. Skryabin, Phys. Rev. A63, 013602 (2001). 